\title{
Uso de TICs para Apoio à Colaboração Durante a COVID-19
}

\author{
Bruna Diirr $^{1,2}$, Tadeu Moreira de Classe ${ }^{1,2}$, Paulo Sérgio Medeiros dos Santos ${ }^{2}$ \\ ${ }^{1}$ Programa de Pós-Graduação em Informática (PPGI) \\ Universidade Federal do Estado do Rio de Janeiro (UNIRIO) \\ Rio de Janeiro - Brasil \\ ${ }^{2}$ Departamento de Informática Aplicada (DIA) \\ Universidade Federal do Estado do Rio de Janeiro (UNIRIO) \\ Rio de Janeiro - Brasil \\ \{bruna.dir, tadeu.classe, pasemes\}euniriotec.br
}

\begin{abstract}
COVID-19 brought changes in the lives of people and organizations. In this context, ICTs play an essential role in maintaining several activities. This research aims to characterize the use of ICTs during the COVID-19 pandemic, analyzing how they support collaborative work in the IT sector. For this, we performed a survey with 368 people, with ICTs being analyzed based on the $3 C$ Collaboration Model besides aspects of perception and collective memory. The results indicate a large and diverse use of ICTs during COVID-19 pandemic as well as support for different dimensions of collaboration, thus offering contributions to Society and CSCW researchers.
\end{abstract}

Resumo. A pandemia da COVID-19 trouxe mudanças na vida das pessoas e organizações. Nesse contexto, as TICs desempenham papel essencial para manutenção de diversas atividades. Esta pesquisa visa caracterizar o uso das TICs durante a pandemia de COVID-19, analisando como elas apoiam o trabalho colaborativo no setor de TI. Para isso, um survey foi realizado com 368 pessoas, sendo as TICs analisadas com base no Modelo 3C de Colaboração, bem como aspectos de percepção e memória coletiva. Os resultados indicam um grande e diverso uso das TICs durante a pandemia de COVID-19, bem como o suporte a diferentes dimensões da colaboração, oferecendo assim contribuições para a Sociedade e comunidade de Sistemas Colaborativos.

\section{Introdução}

A pandemia de COVID-19 ainda é uma realidade em nosso dia-a-dia e seus impactos são percebidos em aspectos que vão além da saúde das pessoas. O grande número de casos e mortes ao redor do mundo e a falta de tratamentos/vacinas para a doença trouxeram mudanças no cotidiano das pessoas (medidas mais severas de higiene, uso de máscaras, fechamento do comércio, distanciamento/isolamento social) que eram impensáveis em outros tempos [Venkatesh e Edirappuli 2020, CDC 2020, Gon et al. 2020, Times 2020]. Instituições de ensino, organizações e indústrias também tiveram que se adaptar a uma nova forma de operação na tentativa de não pararem completamente suas atividades [Shen et al. 2020].

Neste cenário de distanciamento social, as Tecnologias de Informação e Comunicação (TICs) exercem papel essencial no apoio às pessoas em suas atividades 
diárias, permitindo a comunicação entre familiares/amigos, manutenção das atividades de ensino via Internet e continuidade de trabalhos de forma remota [Giles 2020]. Um movimento para continuidade das atividades sociais, acadêmicas e profissionais foi observado em todo mundo, com uso de \#stayAtHome em posts e alteração de valores/quantidade de usos simultâneos de ferramentas [Golby 2020, Giles 2020]. Porém, mesmo em face a essa situação peculiar, ainda pouco se sabe sobre o uso de TICs, especialmente com foco na colaboração, durante a COVID-19.

Este trabalho tem como objetivo investigar o uso e apoio à colaboração por meio das TICs na realização de atividades remotas durante a pandemia de COVID-19. Para isso, foi conduzido um survey para caracterização das atividades realizadas e TICs adotadas nesse período, bem como aspectos colaborativos oferecidos pelas TICs, tendo como participantes profissionais, acadêmicos e estudantes brasileiros do setor de TI. A partir dos resultados da pesquisa, foram observados diferentes perfis, formas como as TICs estão sendo usadas neste período e formas de apoio ao trabalho colaborativo.

Assim, as seções deste artigo são dispostas da seguinte forma. A seção 2 apresenta o contexto da pesquisa, explicando o papel da colaboração durante crises, como na COVID-19. A visão sobre aspectos de colaboração e como eles se encaixam na pesquisa é apresentada na seção 3. A seção 4 detalha a metodologia da pesquisa, descrevendo questões, abordagens, etapas e como o survey foi projetado. A seção 5 apresenta a estratificação da análise dos dados e resultados das respostas dos participantes. A seção 6 discute os achados da pesquisa. E, finalmente, a seção 7 aponta as conclusões do artigo.

\section{Contexto da Pesquisa: Colaboração em Eventos Perigosos ou Impactantes}

Pesquisas relacionadas à COVID-19 requerem o entendimento sobre à dinâmica de eventos perigosos. Neste contexto, diferentes termos são identificados: crise, emergência, desastre. Uma crise é um evento anormal, com altos riscos e intensas dificuldades, em que as habilidades das pessoas para gerenciarem atividades diminuem drasticamente [Sawalha 2014]. Emergências surgem da falha ao controlar uma crise, indicando um evento crítico e inesperado que requer atenção imediata para evitar consequências negativas [Sawalha 2014, Donnelly e Proctor-Thomson 2015, Vivacqua et al. 2016]. Emergências podem terminar em desastres: um evento que causa disrupção social, excedendo a capacidade de dada comunidade para lidar com ele, além de demandar enorme esforço para restaurar a situação a um estado que pode ser controlado [Sawalha 2014]. Independente do termo, esses eventos "perigosos ou impactantes" dizem respeito ao inter-relacionamento entre sociedade (indivíduos, grupos, comunidades e organizações), ambiente físico (conexões sociais afetadas) e cultura (normas e valores) [Semaan e Mark 2012]. Tsunamis, furacões, terremotos etc., são lembranças constantes da natureza destrutiva/imprevisível de tais eventos e da importância de estudar o tema [Donnelly e Proctor-Thomson 2015, Vivacqua et al. 2016].

Nesse contexto em que as pessoas precisam trabalhar em sintonia para lidarem com mudanças frequentes, sobrecarga de informações não confirmadas/incompletas/ imprecisas/conflitantes e resultados inesperados de decisões, a colaboração se torna um elemento chave [Vivacqua et al. 2016]. Ela melhora a resiliência das pessoas afetadas, facilita ações de resposta e recuperação e ajuda a reduzir ou minimizar o impacto de consequências adversas [Sawalha 2014]. Oportunidades do uso de TICs para apoiar a 
colaboração nesse contexto também são evidentes [Pipek et al. 2012]. Tais tecnologias permitem que um grande número de pessoas coordenem esforços para disseminar informações relevantes, determinem a segurança de entes queridos, mantenham suas rotinas sociais/laborais/educacionais, e "reimaginem" a própria sociedade e práticas em eventos com efeitos de longa duração [Semaan e Mark 2012, Vivacqua et al. 2016].

[Semaan e Mark 2012] apontam que várias pesquisas investigaram como as TICS apoiam as pessoas a lidarem, se adaptarem e retornarem às atividades em eventos perigosos. Porém, estes autores vão além, focando sua pesquisa em eventos em que as pessoas sofrem impactos prolongados e precisam se adaptar e conviver com constantes incertezas no ambiente - como em guerras, onde eventos aleatórios ocorrem diariamente e podem acontecer a qualquer hora, em qualquer lugar - destacando como as TICs permitem que as pessoas mantenham contato social. Estamos enfrentando um cenário similar durante a pandemia da COVID-19, uma vez que vacina ainda estão em distribuição e as pessoas estão em risco de contaminação apenas por saírem de casa.

[Donnelly e Proctor-Thomson 2015] também argumentam que os desastres impactam as atividades laborais, pois operar em ambientes de incerteza promove uma reavaliação das prioridades das pessoas/organizações/sociedade e leva à adoção de novas formas de trabalho, como o teletrabalho. O teletrabalho fornece meios para restaurar e garantir a capacidade operacional em desastres, já que facilita a redistribuição de tarefas pela força de trabalho quando os locais de trabalho estão inacessíveis/inseguros e garante a continuidade de atividades críticas/essenciais. Estes movimentos de trabalho/estudo remotos também são observados durante a COVID-19, sendo inicialmente descritos por [Belzunegui-Eraso e Erro-Garcés 2020, Dubey e Tripathi 2020, Owusu-Fordjour et al. 2020].

Os trabalhos mencionados acima discutem aspectos relacionados a esta pesquisa, como eventos de longa duração e impacto no trabalho das pessoas. Porém, eles detalham (a) o uso de uma única tecnologia (Facebook) para manter a interação social, ignorando outras atividades como educação e trabalho [Semaan e Mark 2012]; (b) o impacto de um desastre específico (terremoto) no trabalho das pessoas, ignorando eventos com efeitos de longa duração [Donnelly e Proctor-Thomson 2015]; e (c) pesquisas sobre trabalho e educação remota durante a COVID-19, mas que não focam no apoio colaborativo usando TICs [Belzunegui-Eraso e Erro-Garcés 2020, Dubey e Tripathi 2020, Owusu-Fordjour et al. 2020, Zhang et al. 2020]. Neste sentido, entende-se que ainda não há uma análise aprimorada sobre como as pessoas estão usando tecnologias colaborativas para manterem suas atividades durante a pandemia de COVID-19. Argumenta-se que uma análise durante a pandemia de COVID-19 ajudaria a entender quais TICs são usadas e capazes de fornecer apoio colaborativo às pessoas em eventos impactantes e, adicionalmente, como as pessoas estão usando tais TICs para colaborarem em eventos específicos.

\section{Visão da Abordagem Colaborativa para a Pesquisa}

A presente pesquisa nasceu da percepção que as atividades diárias vem sendo realizadas de forma alternativa devido a mudanças abruptas na rotina da sociedade [Levy 2010]. O distanciamento social representa um caso extremo, sendo necessário compreender em que extensão a atual infraestrutura tecnológica pode oferecer suporte durante eventos 
com efeitos de longa duração de forma que as pessoas possam manter suas atividades sociais/laborais/educacionais. Desta forma, argumenta-se que este trabalho fornece interessante contribuição para o campo de estudo e prática em Sistemas Colaborativos.

O Modelo 3C de Colaboração [Fuks et al. 2008] foi usado para desenvolver questões, coletar e interpretar dados sobre o objetivo da pesquisa. Este modelo analisa a colaboração sob a ótica de três dimensões: (a) Comunicação, que possibilita a troca de mensagens, argumentação e negociação entre as pessoas envolvidas na colaboração; (b) Coordenação, que inclui o gerenciamento e controle da colaboração, considerando as pessoas envolvidas e como elas interagem, executam atividades e usam recursos em um espaço compartilhado; e (c) Cooperação, que compreende o trabalho que as pessoas desenvolvem em conjunto no espaço compartilhado (Figura 1). Esse modelo também destaca a Percepção, que emerge como consequência da aplicação das outras três dimensões e envolve mecanismos que permitem saber "quem é responsável pelo quê, em que tempo e onde", bem como o impacto que dada atividade terá sobre o trabalho dos demais membros do grupo. Além disso, é importante mencionar que a colaboração possibilita a troca de conhecimento pelo grupo, gerando assim uma Memória Coletiva que pode ser consultada e utilizada pelos membros do grupo sempre que necessário [Fuks et al. 2008][Mistrík et al. 2010]

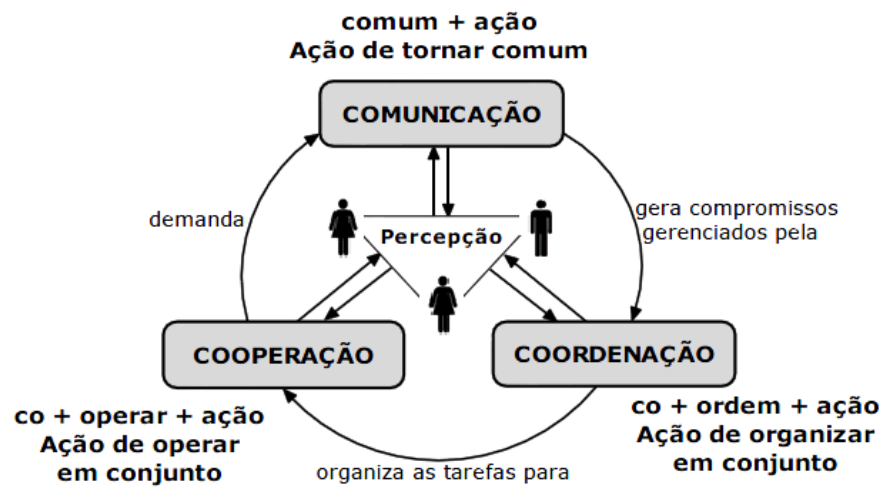

Figura 1. Modelo 3C

\section{Metodologia de Pesquisa}

Uma pesquisa baseada em survey foi realizada. Surveys possibilitam agrupar grande quantidade de pessoas para responder um mesmo conjunto de questões, oferecendo também outros benefícios como uniformidade de respostas, anonimização de participantes e possibilidade de aplicação remota (requisito fundamental neste período de COVID-19) [McMillan e Schumacher 2010]. A realização do survey baseou-se nos guias definidos por [Kasunic 2005], o qual é apoiado pela abordagem GQM (Goal-Question-Metric) [Basili 1992] (Figura 2), que auxiliou a definição do objetivo da pesquisa, derivação de questões de análise e métricas e interpretação dos dados.

$\mathrm{Na}$ etapa de definição, o objetivo da pesquisa foi identificado seguindo a abordagem GQM: analisar Tecnologias de Informação e Comunicação; com $o$ propósito de caracterização; no que diz respeito a aspectos colaborativos; do ponto de vista de profissionais, acadêmicos e estudantes de TI; no contexto da continuidade 


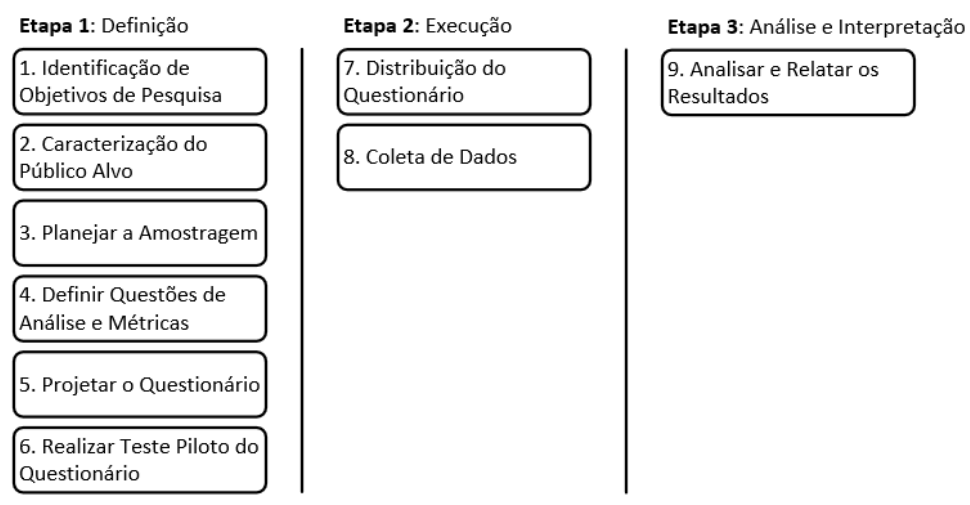

Figura 2. Processo de realização do survey (baseado em [Kasunic 2005])

de trabalho/estudo durante o distanciamento social da COVID-19. Em seguida, o público-alvo foi planejado, compreendendo profissionais, professores e estudantes que atuam no setor de TI. Este público foi escolhido devido à possibilidade de trabalho em home-office para manter as atividades nas organizações e a adesão de vários cursos de bacharelado/licenciatura/tecnólogos à modalidade de ensino não presencial para prosseguirem com aulas e atividades junto aos alunos.

Com o objetivo determinado, foi definida a questão de pesquisa: "Quais TICs e que tipos de colaboração estão sendo desempenhadas para manutenção das atividades de profissionais, professores e estudantes de TI durante a pandemia de COVID-19?". Também foi construído um questionário para coletar as opiniões dos participantes sobre TICs usadas, atividades desempenhadas e aspectos colaborativos (Modelo 3C, percepção e memória coletiva) percebidos nestas tecnologias (Tabela 1). O questionário foi disponibilizado via Google Forms ${ }^{1}$ e, por meio de um teste piloto, suas questões foram avaliadas e ajustadas para melhorar a coleta e análise de dados [Coelho et al. 2019].

$\mathrm{Na}$ etapa de execução, o questionário foi compartilhado por meio das mídias sociais e e-mail dos autores. Na expectativa de aumentar a taxa e heterogeneidade de respostas, um convite também foi enviado para outras listas de e-mail (SBC e de universidades), solicitando amplo compartilhamento. As respostas foram aceitas durante 11 dias, entre 10/04/2020 e 21/04/2020. Ao final, foram obtidas 391 respostas, dentre as quais 368 foram consideradas válidas. A exclusão de 23 participantes aconteceu devido ao relato de não utilização de TICs durante o período de isolamento social ou não atuação no setor de TI (com exceção dos estudantes).

$\mathrm{Na}$ etapa de análise e interpretação, todos os dados foram analisados utilizando os softwares $R$ Statistics 3.6.1 e Microsoft Excel 2019". Todas as informações foram extraídas exclusivamente das respostas coletadas. A análise dos dados foi guiada visando responder a questão principal da pesquisa com base nos dados coletados ${ }^{2}$.

\subsection{Ameaças à Validade do Estudo}

Estudos baseados em survey podem estar associados a várias ameaças de validade. As ameaças de validade interna (i.e., como os achados apoiam as afirmações sobre o

\footnotetext{
${ }^{1}$ Google Forms: https://www.google.com/forms

${ }^{2}$ Dados: https://bit.ly/TI-COVID-Dataset
} 
Tabela 1. Estrutura de questionário usado no survey

\begin{tabular}{|c|c|c|}
\hline Seções & Questão & Tipo de Resposta \\
\hline $\begin{array}{c}\text { Descrição e } \\
\text { consentimento } \\
\text { (1 questão) }\end{array}$ & Aceita participar desta pesquisa? & Duas opções (excludentes) \\
\hline \multirow{2}{*}{$\begin{array}{l}\text { Isolamento } \\
\text { social } \\
\text { (2 questões) }\end{array}$} & $\begin{array}{l}\text { Como classifica sua situação atual de } \\
\text { isolamento? }\end{array}$ & Múltipla escolha (excludente) \\
\hline & $\begin{array}{l}\text { Está fazendo uso de TICs para continuidade } \\
\text { de suas atividades? }\end{array}$ & Duas opções (excludentes) \\
\hline \multirow{2}{*}{$\begin{array}{l}\text { Uso de } \mathbf{T I C s} \\
\text { (2 questões) }\end{array}$} & $\begin{array}{l}\text { Para que propósito você está usando as } \\
\text { tecnologias selecionadas? }\end{array}$ & $\begin{array}{l}\text { Múltipla escolha (não excludente) } \\
\text { Grade de seleção (TICs x Atividades) }\end{array}$ \\
\hline & $\begin{array}{l}\text { Informe outras TICs e/ou outras atividades } \\
\text { diferentes das listadas na questão anterior. }\end{array}$ & Discursiva (opcional) \\
\hline \multirow{5}{*}{$\begin{array}{l}\text { Colaboração } \\
\text { (5 questões) }\end{array}$} & $\begin{array}{l}\text { Quais das TICs listadas estão sendo usadas } \\
\text { para a Comunicação com outras pessoas? }\end{array}$ & Múltipla escolha (não excludente) \\
\hline & $\begin{array}{l}\text { Quais das TICs listadas estão sendo usadas } \\
\text { para a Coordenação de atividades? }\end{array}$ & Múltipla escolha (não excludente) \\
\hline & $\begin{array}{l}\text { Quais das TICs listadas estão sendo usadas } \\
\text { para a Cooperação com outras pessoas? }\end{array}$ & Múltipla escolha (não excludente) \\
\hline & $\begin{array}{l}\text { Quais das TICs listadas estão sendo usadas } \\
\text { para o acompanhamento ou Percepção das } \\
\text { atividades realizadas pelas pessoas? }\end{array}$ & Múltipla escolha (não excludente) \\
\hline & $\begin{array}{l}\text { Quais das TICs listadas estão sendo usadas } \\
\text { para a Gerenciar o Conhecimento (Memória } \\
\text { Social)? }\end{array}$ & Múltipla escolha (não excludente) \\
\hline \multirow{7}{*}{$\begin{array}{c}\text { Perfil do } \\
\text { Participante } \\
\text { (7 questões) }\end{array}$} & Gênero & Múltipla escolha (excludente) \\
\hline & Faixa etária & Múltipla escolha (excludente) \\
\hline & Estado de residência & Múltipla escolha (excludente) \\
\hline & Formação acadêmica & Múltipla escolha (excludente) \\
\hline & Profissão (mercado, professor, estudante) & Múltipla escolha (não excludente) \\
\hline & Descrição da profissão (para mercado) & Discursiva \\
\hline & E-mail & Discursiva (opcional) \\
\hline
\end{tabular}

objeto de estudo) se concentram principalmente no design do questionário, em como os participantes irão respondê-los, e na análise dos dados. Um estudo piloto foi conduzido visando a compreensão das questões, detecção de dificuldades, e identificação de problemas que não puderam ser antecipados. Além disso, foram consideradas as escalas mais adequadas ao longo do design do questionário para minimizar ameaças de conclusão associadas à escala usada.

Sobre as ameaças de validade externa (i.e., se as conclusões podem ser aplicadas a outros ambientes), a representatividade dos participantes desempenha um papel crucial. Apesar de ter sido adotada a técnica de seleção por conveniência, que pode levar a uma amostragem indesejavelmente homogênea, técnicas como snowballing e agrupamento por desvios padrão sobre as variáveis do perfil do participante foram usadas para obter amostras mais heterogêneas. Também é possível que haja um viés nas TICs incluídas no survey. Os autores optaram pela inclusão de TICs conhecidas e/ou utilizadas por eles, porém permitiram que os participantes enumerassem TICs adicionais. Além disso, os resultados podem ser influenciados por questões relacionadas a configurações regionais.

\section{Análise dos Dados e Resultados}

\subsection{Perfil dos Participantes}

O estudo obteve a participação de pessoas de 18 dos 26 estados do Brasil, além do Distrito Federal. Também foram observadas respostas de 4 participantes brasileiros que vivem no exterior (Figura 3). Os estados do sudeste concentraram a maior parte das respostas $(81 \%)$. Esta participação desbalanceada pode estar relacionada à seleção por 
conveniência, uma vez que os autores do trabalho residem nesta região, mas também por ser sabido que a região sudeste concentra quase metade da população brasileira e abriga a maioria das instituições de ensino e empresas de TI.

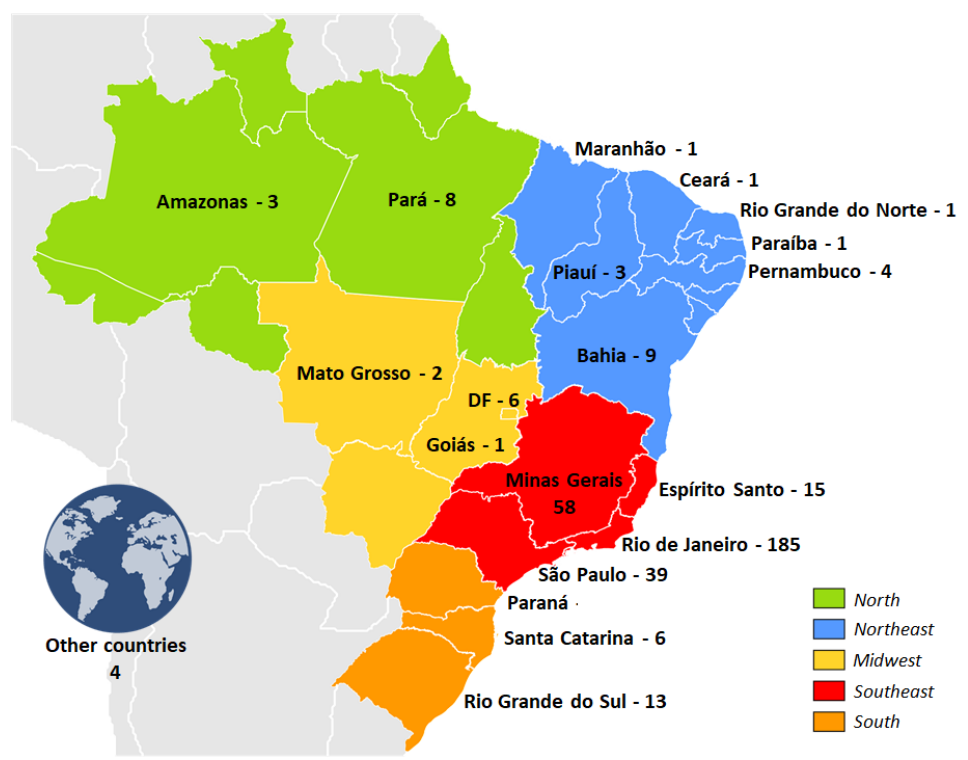

Figura 3. Distribuição dos participantes

Outras informações, como gênero, idade, formação acadêmica e ocupação, também foram coletadas (Tabela 2). Com respeito a ocupação, os participantes puderam selecionar mais de uma opção (ex.: estudantes e professores, também podem ser profissionais ativos no mercado de TI). Os dados mostram uma participação significativa de jovens adultos, do sexo masculino e com ensino superior. A maior participação do sexo masculino já era esperada, uma vez que eles representam a maioria dos profissionais, estudantes e acadêmicos no setor de TI do país [Nunes 2015]. Ademais, a alta participação de pessoas com formação superior reflete o público-alvo deste estudo.

Tabela 2. Perfil dos Participantes

\begin{tabular}{|c|c|c|c|c|c|c|c|}
\hline \multirow{2}{*}{\multicolumn{2}{|c|}{ Variáveis }} & \multicolumn{2}{|c|}{ Total } & \multirow{2}{*}{\multicolumn{2}{|c|}{ Variáveis }} & \multicolumn{2}{|c|}{ Total } \\
\hline & & \multirow{2}{*}{$\frac{\text { Qtde. }}{244}$} & \multirow{2}{*}{\begin{tabular}{|c|}
$\%$ \\
$66 \%$ \\
\end{tabular}} & & & Qtde. & $\%$ \\
\hline \multirow{3}{*}{ Gênero } & Masculino & & & \multirow{6}{*}{ Formação } & Ensino Fundamental & 1 & $0 \%$ \\
\hline & Feminino & 121 & $33 \%$ & & Ensino Médio & 22 & $6 \%$ \\
\hline & Outros & 3 & $1 \%$ & & Ensino Superior & 104 & $28 \%$ \\
\hline \multirow{7}{*}{$\begin{array}{l}\text { Faixa } \\
\text { Etária }\end{array}$} & $00-19$ & 8 & $2 \%$ & & Especialização & 47 & $13 \%$ \\
\hline & $20-29$ & 103 & $28 \%$ & & Mestrado & 79 & $21 \%$ \\
\hline & $30-39$ & 112 & $30 \%$ & & Doutorado & 115 & $31 \%$ \\
\hline & $40-49$ & 76 & $21 \%$ & \multirow{7}{*}{ Ocupação } & \begin{tabular}{|l} 
Estudante \\
\end{tabular} & 51 & $14 \%$ \\
\hline & $50-59$ & 56 & $15 \%$ & & Professor & 120 & $33 \%$ \\
\hline & $60-69$ & 10 & $3 \%$ & & Profissional & 84 & $23 \%$ \\
\hline & $70+$ & 3 & $1 \%$ & & Professor e Estudante & 11 & $3 \%$ \\
\hline & & & & & Professor e Profissional & 29 & $8 \%$ \\
\hline & & & & & Estudante e Profissional & 55 & $15 \%$ \\
\hline & & & & & Professor, Estudante e Profissional & 18 & $5 \%$ \\
\hline
\end{tabular}

\subsection{Suporte à Colaboração}

Inicialmente, o survey listou 21 TICs. Para ajudar a analisar os dados, cada uma destas TICs foram categorizadas em 7 grupos, de acordo com o propósito original pela qual ela 
foi desenvolvida (Tabela 3). Por exemplo: o Google Meet foi categorizado dentro do grupo de reuniões, uma vez que esse é seu objetivo principal. É importante mencionar que esta classificação não foi usada no questionário para não inserir um viés, e influenciar na resposta dos participantes ao selecionar a forma com que eles têm usado tais TICs.

Tabela 3. Categorização das TICs para análise

\begin{tabular}{|c|l|}
\hline Categoria & \multicolumn{1}{|c|}{ TICs } \\
\hline Reuniões & Skype, Zoom, Google Meet/Hangouts/Duo, Microsoft Teams, Webex Meetings \\
\hline Mensagens & E-mail, WhatsApp, Telegram, Discord, Slack \\
\hline Documentos & Google Docs, Microsoft Office 365, Overleaf Latex \\
\hline Coordenação de Tarefas & Trello \\
\hline Educação & Google Classroom, Moodle \\
\hline Armazenamentos & Dropbox, Google Drive, One Drive \\
\hline Outros & Facebook, Twitter \\
\hline
\end{tabular}

Desta maneira, a Figura 4 apresenta a distribuição das TICs, listadas no questionário e sugeridas pelos participantes, em relação ao suporte a cada dimensão colaborativa que consideramos no estudo.

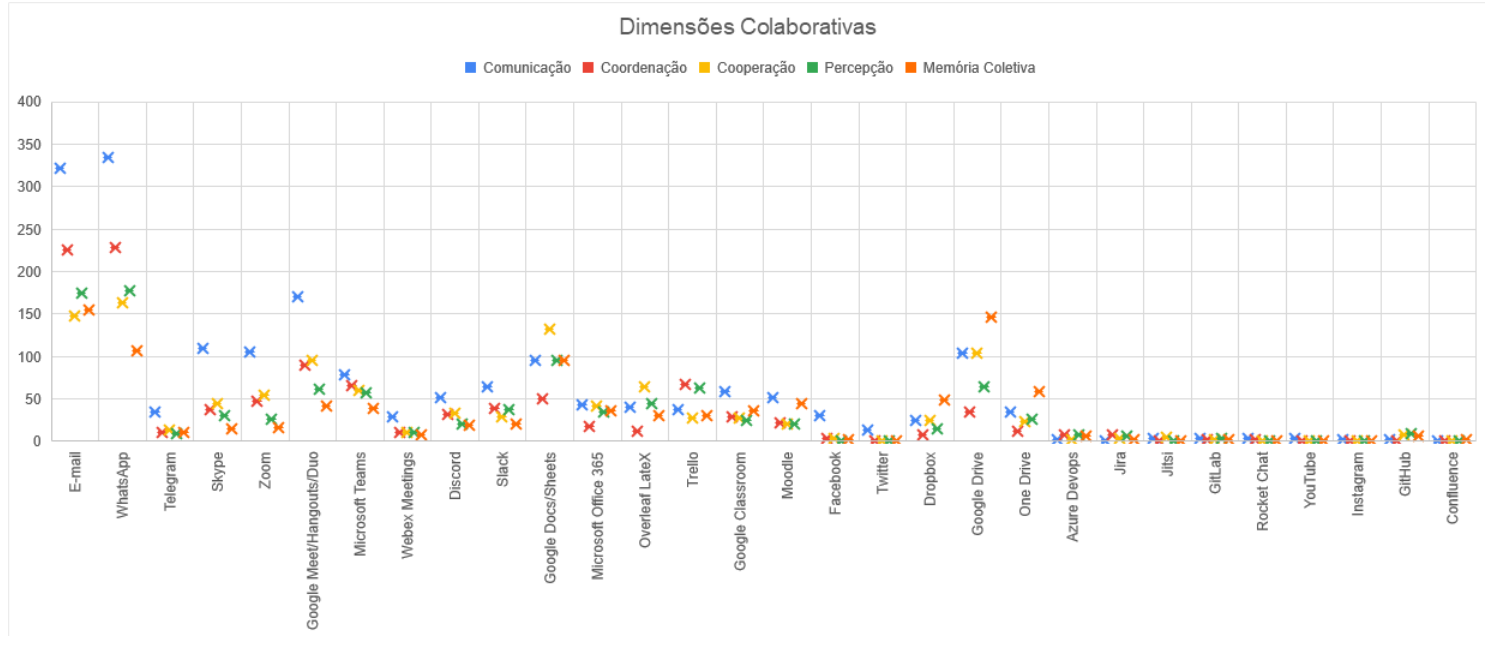

Figura 4. TICs e suporte às dimensões colaborativas

\subsubsection{Comunicação}

Os resultados não apresentaram surpresas em relação à dimensão de comunicação. Como é possível perceber na Figura 4, as TICs mais usadas pelos participantes foram as relacionadas às categorias mensagens (WhatsApp - 344 menções; E-Mail - 322 menções) e reuniões (Google Meet/Hangouts/Duo - 170 menções; Skype - 109 menções; Zoom 105 menções). Estas categorias estão intimamente ligadas ao objetivo dessa dimensão da colaboração (possibilitar troca de mensagens, argumentações e negociações entre as pessoas envolvidas). Também é possível observar o suporte à comunicação em TICs de outras categorias, tais como armazenamento e documentos, tendo as maiores menções o Google Drive (104) e o Google Docs/Sheets (96 menções). Estes resultados indicam que a comunicação entre os participantes não ocorre somente pela troca de mensagens explícitas 
e pode (e deve) ser feita também por outros meios, como documentos e repositórios que armazenam informações importantes para a argumentação e negociação entre as pessoas.

\subsubsection{Coordenação}

Para a dimensão de coordenação, as TICs mais usadas pelos participantes são aquelas voltadas à troca de mensagens (WhatsApp - 228 menções; E-mail - 226 menções) e reuniões (Google Meet/Hangouts/Duo - 89 menções; Microsoft Teams - 65 menções). A categoria "Coordenação de Tarefas", a qual mais se aproxima do objetivo deste aspecto colaborativo (gestão e controle da colaboração), obteve apenas 67 respostas. Uma possível causa para este número pode ter sido a inclusão de uma única TIC no questionário (Trello). Porém, é importante ressaltar que 47 participantes citaram o uso de outras ferramentas de coordenação, tais como: GitLab (12 menções), GitHub (10 menções), Azure DevOps (6 menções), Jira (5 menções) e Doodle (3 menções). A Figura 4 também permite observar um grande uso de TICs de outras categorias (troca de mensagens e reuniões, principalmente), indicando que a coordenação da colaboração muitas vezes é operacionalizada através do uso de outras TICs que não foram projetadas para esta natureza. As pessoas podem usar tais tecnologias para se comunicarem sobre como gerenciar e controlar as atividades do grupo, realizando ações para este fim de formas diferentes (com ou sem TICs desenvolvidas com o propósito de coordenação).

\subsubsection{Cooperação}

Para a dimensão de cooperação, como pode ser observado na Figura 4, as TICs mais usadas pelos participantes foram aquelas das categorias de mensagens (WhatsApp 163 menções; E-Mail - 148 menções), edição de documentos (Google Docs/Sheet 132 menções), armazenamento (Google Drive - 104 menções) e reuniões (Google Meet/Hangouts/Duo - 96 menções). As categorias documentos e armazenamento estão intimamente relacionadas ao objetivo desta dimensão colaborativa (o trabalho que um grupo desenvolve em conjunto). Porém, como percebido na dimensão de coordenação, a grande indicação de TICs na categoria de troca de mensagens e reuniões também pode indicar pessoas fazendo uso de outras tecnologias, que não aquelas desenvolvidas para o propósito de cooperação, para comunicar como um trabalho conjunto deve ser realizado.

\subsubsection{Percepção}

Sobre a dimensão da percepção (Figura 4), os participantes indicaram as TICs das categorias mensagens (WhatsApp - 177 menções; E-Mail - 175 menções), documentos (Google Docs/Sheet - 96 menções), armazenamento (Google Drive - 64 menções), coordenação de tarefas (Trello - 63 menções) e reuniões (Google Meet/Hangouts/Duo - 62 menções; Microsoft Teams - 57 menções). Como a percepção se origina das outras dimensões de colaboração, os participantes podem entender que diferentes TICs oferecem mecanismos que permitem saber "quem é responsável pelo quê", "em quem momento" e "onde", bem como tarefas que podem impactar outras atividades. 


\subsubsection{Memória Coletiva}

Em relação à memória coletiva, a Figura 4 mostra que os participantes indicaram TICs informadas guiadas pela troca de mensagens (E-Mail - 155 menções; WhatsApp - 106 menções), armazenamento (Google Drive - 146 menções) e edição de documentos (Google Docs/Sheet - 95 menções). Estas categorias estão intimamente relacionadas ao objetivo da memória coletiva, onde os membros do grupo podem criar e consultar conhecimento necessário para a colaboração. Além disso, é importante dizer que existe suporte para memória coletiva em TICs classificadas em outras categorias.

\section{Discussão}

O objetivo desde estudo foi caracterizar como as TICs estão sendo usadas para a colaboração, considerando o cenário de isolamento social durante a pandemia de COVID-19. Com esta categorização, argumenta-se que o trabalho mostra que: i) uma porção significativa de participantes podem executar suas tarefas usuais durante o distanciamento social; ii) as TICs estão, de fato, oferecendo suporte à colaboração dentro deste cenário de evento perigoso.

O survey demonstrou que o uso de TICs durante a COVID-19 é consistente entre diferentes perfis demográficos, derivados de todo o Brasil e analisados neste estudo. Baseando-se no Modelo 3C de Colaboração e nos aspectos de percepção e memória coletiva, a resposta principal à questão de pesquisa deste artigo é que os participantes estão usando as TICs, majoritariamente para suporte à comunicação e cooperação com seus pares. Isso se tornou mais claro a partir da observação do uso de TICs para troca de mensagens, reuniões e edição de documentos. Além disso, também ressalta que as outras dimensões colaborativas são importantes para que os participantes mantenham seus trabalhos. Sem coordenação, tarefas não podem ser atribuídas ou gerenciadas; sem percepção torna-se desafiador compreender responsabilidades, onde uma tarefa é realizada e seu impacto em outras atividades; e sem memória compartilhada, o histórico de colaboração ou contexto sobre uma decisão tomada é perdido.

Também é importante destacar o uso de TICs específicas, tais como WhatsApp e E-Mail, pelos participantes em todas as dimensões colaborativas. O amplo uso destas TICs pode ser explicado pela difusão de tais ferramentas para uso pessoal na cultura brasileira, a qual, devido à necessidade do trabalho remoto, são usadas para fins profissionais com maior frequência.

\section{Conclusões}

A COVID-19 já é um marco na história mundial: levou à uma pandemia global, que infelizmente trouxe inúmeras mortes e mudanças na vidas das pessoas devido ao isolamento necessário para controlar a doença. Neste cenário, as TICs têm desempenhado um importante papel para manutenção das atividades sociais, laborais e educacionais das pessoas durante este período de isolamento. Entretanto, não havia informação mais detalhada sobre como as pessoas estão usando tais tecnologias e como elas estão sendo utilizadas como foco no trabalho colaborativo.

Este artigo apresentou um survey que visa caracterizar o uso de TICs durante a pandemia de COVID-19, em relação às suas dimensões colaborativas, do ponto de 
vista de trabalhadores, estudantes e acadêmicos brasileiros do setor de TI. Os resultados mostraram que diferentes perfis foram afetados pelos distanciamento social, mas que conseguiram continuar suas atividades através do trabalho remoto apoiado por TICs. Foi possível perceber as TICs mais usadas, para qual fim e as dimensões colaborativas suportadas.

Assim, acredita-se que os resultados deste survey possam trazer benefícios para a sociedade, especialmente para as pessoas do setor de TI, mostrando quais TICs foram mais usadas e as dimensões colaborativas que elas podem dar suporte. Contudo, existem algumas limitações do estudo que merecem ser mencionadas: i) o estudo focou na análise da contagem de respostas dos participantes para descrever e caracterizar os aspectos colaborativos e responder a questão de pesquisa; ii) uma perspectiva qualitativa de análise de discurso e respostas discursivas podem adicionar uma descrição mais robusta dos achados apresentados neste artigo.

Como trabalho futuro, espera-se realizar um survey com pessoas que não são do setor de TI, visando explorar aspectos que podem não ter sido observados no público deste trabalho. Adicionalmente, gostaríamos de realizar novamente a pesquisa com pessoas do setor de TI, após um período de tempo, para verificar se houveram alterações no uso das TICs e como elas foram utilizadas.

\section{Referências}

Basili, V. R. (1992). Software modeling and measurement: the goal/question/metric paradigm. Technical report.

Belzunegui-Eraso, A. e Erro-Garcés, A. (2020). Teleworking in the context of the covid-19 crisis. Sustainability, 12(9):3662.

CDC (2020). Use of cloth face coverings to help slow the spread of covid-19. Centers for Disease Control and Prevention. Disponível em: <https://cutt.ly/DfYdulW $>$. Acessado em 09 de setembro de 2020.

Coelho, J., Souza, G. H., e Albuquerque, J. (2019). Desenvolvimento de questionários e aplicação na pesquisa em informática na educação. Metodologia de Pesquisa em Informática na Educação: Abordagem Quantitativa de Pesquisa. Porto Alegre: SBC. Série Metodologia de Pesquisa em Informática na Educação, 2.

Donnelly, N. e Proctor-Thomson, S. B. (2015). Disrupted work: home-based teleworking (hbtw) in the aftermath of a natural disaster. New Technology, Work and Employment, 30(1):47-61.

Dubey, A. D. e Tripathi, S. (2020). Analysing the sentiments towards work-from-home experience during covid-19 pandemic. Journal of Innovation Management, 8(1).

Fuks, H., Raposo, A., Gerosa, M. A., et al. (2008). The 3c collaboration model. In Encyclopedia of E-collaboration, pages 637-644. IGI Global.

Giles, M. (2020). Free software that businesses, schools and others can use during the covid-19 crisis. Forbes. Disponível em: <https://cutt.ly/XfR6Ldz>. Acessado em 9 de setembro de 2020.

Golby, J. (2020). \#stayathome. Planning News, 46(5):16. 
Gon, G., Dancer, S., Dreibelbis, R., Graham, W. J., e Kilpatrick, C. (2020). Reducing hand recontamination of healthcare workers during covid-19. Infection Control \& Hospital Epidemiology, pages 1-2.

Kasunic, M. (2005). Designing an effective survey. Technical report, Carnegie-Mellon Univ Pittsburgh PA Software Engineering Inst.

Levy, P. (2010). Cibercultura. Editora 34.

McMillan, J. H. e Schumacher, S. (2010). Research in education: Evidence-based inquiry, myeducationlab series. Pearson.

Mistrík, I., Grundy, J., Van der Hoek, A., e Whitehead, J. (2010). Collaborative software engineering: challenges and prospects. In Collaborative software engineering, pages 389-403. Springer.

Nunes, D. (2015). Educação superior em computação, estatísticas 2015. Sociedade Brasileira de Computação. Disponível em: <https://cutt.ly/vfG1LPe > Acessado em 18 de setembro de 2020.

Owusu-Fordjour, C., Koomson, C., e Hanson, D. (2020). The impact of covid-19 on learning-the perspective of the ghanaian student. European Journal of Education Studies.

Pipek, V., Palen, L., e Landgren, J. (2012). Workshop summary: collaboration \& crisis informatics (cci'2012). In Proceedings of the ACM 2012 conference on Computer Supported Cooperative Work Companion, pages 13-14.

Sawalha, I. H. S. (2014). Collaboration in crisis and emergency management: Identifying the gaps in the case of storm 'alexa'. Journal of Business Continuity \& Emergency Planning, 7(4):312-323.

Semaan, B. e Mark, G. (2012). 'facebooking'towards crisis recovery and beyond: disruption as an opportunity. In Proceedings of the ACM 2012 conference on computer supported cooperative work, pages 27-36.

Shen, S., Chen, O., Gao, A., Mok, L., Sun, J., e Wan, D. D. (2020). Coronavirus (covid-19) outbreak: Short- and long-term actions for cios. Gartner Reprint. Disponível em: $<$ https://www.gartner.com/doc/reprints?id=1-1YNHRJU4\&ct=200319>. Acessado em 9 de setembro de 2020 .

Times, L. A. (2020). Coronavirus social distancing around the world. Los Angeles Times. Disponível em: <https://cutt.ly/ZfR5KDl>. Acessado em 9 de setembro de 2020.

Venkatesh, A. e Edirappuli, S. (2020). Social distancing in covid-19: what are the mental health implications? Bmj, 369.

Vivacqua, A., Garcia, A. C., Canós, J., Comes, M., e Vieira, V. (2016). Collaboration and decision making in crisis situations. In Proceedings of the 19th ACM Conference on Computer Supported Cooperative Work and Social Computing Companion, pages 503-508.

Zhang, W., Wang, Y., Yang, L., e Wang, C. (2020). Suspending classes without stopping learning: China's education emergency management policy in the covid-19 outbreak. 